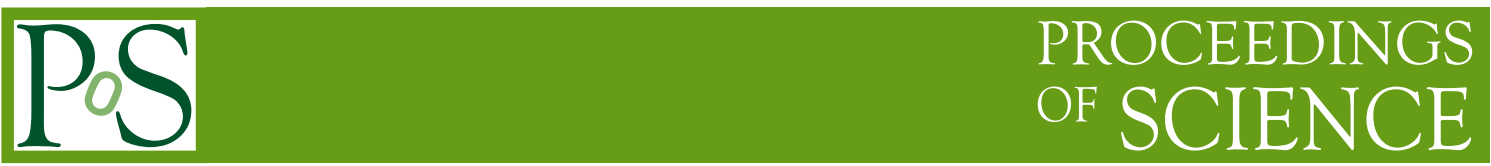

\title{
Neutrino mass hierarchy with PINGU
}

\author{
Sebastian Böser* for the PINGU-IceCube collaboration ${ }^{\dagger}$ \\ Universität Mainz, Germany \\ E-mail: sboeser@uni-mainz.de
}

\begin{abstract}
Neutrino oscillations - first measured via atmospheric neutrinos - have so far provided the only current direct evidence for physics beyond the Standard Model of Elementary Particles. The mixing angles and mass-squared differences that describe oscillations in the neutrino sector have been measured with high precision through the efforts of a variety of experiments worldwide [1], with the exception of the charge-parity phase and the ordering of the mass eigenstates (the neutrino mass hierarchy - NMH). The low-energy in-fill DeepCore to the IceCube detector array at the geographic South Pole provides sensitivity to the oscillations of atmospheric neutrinos with an unprecedented effective detector mass. An analysis of the mixing parameters $\theta_{23}$ and $\Delta m_{32}^{2}$ based on three years of data yields precision comparable to other leading experiments. Building on this success and benefiting from a relatively large value of the mixing-angle between the first and third mass eigenstate states, the Precision IceCube Next-Generation Upgrade (PINGU) is aiming at measuring the mass hierarchy via atmospheric neutrinos using a very large volume iceCherenkov detector. Further increasing the photocathode density in the DeepCore region permits a lowering of the energy threshold in the fiducial volume to the region that is affected by MSW and parametric oscillations [2,3], and thus extraction of the hierarchy. Here we discuss DeepCore results on atmospheric mixing parameters, the design of the PINGU detector and its sensitivity to the mass hierarchy and $v_{\tau}$ appearance.
\end{abstract}

XVI International Workshop on Neutrino Telescopes,

2-6 March 2015

Palazzo Franchetti - Istituto Veneto, Venice, Italy

\footnotetext{
*Speaker.

${ }^{\dagger}$ http://www.icecube.wisc.edu
} 


\section{Introduction}

In the 1990s, Super-Kamiokande's measurements of atmospheric neutrinos [4] led to the acceptance of the mass-induced oscillation model where neutrinos are massive particles whose interaction eigenstates are not their mass eigenstates. This property gives neutrinos produced in one flavor eigenstate, $\alpha$, a non-zero probability of interacting in a different flavor, $\beta$, after traveling for some distance $L$. In the simplest scenario, with only two neutrino flavors, the transition probability from state $\alpha$ to state $\beta$ is given by

$$
P\left(v_{\alpha} \rightarrow v_{\beta}\right)=\sin ^{2}(2 \theta) \sin ^{2}\left(\Delta m^{2} \frac{L}{4 E_{v}}\right),
$$

where $\theta$ defines the mixing amplitude between flavor eigenstates, $\Delta m^{2}$ is the difference in the squared masses and $E_{v}$ is the neutrino energy (all in natural units). Considering the existence of three neutrino flavors provides an oscillation probability that consists of a sum of terms of the form of Eq. 1.1, but involving three mixing angles, two mass-squared differences and a complex phase describing CP-violation. Currently, the mixing angles, the solar mass splitting and the absolute value of the atmospheric mass splitting have been measured [1] while the existence of $\mathrm{CP}$-violation and the ordering of the masses remain open questions [5, 1]. Addressing these questions requires improving the measurement precision on the known parameters as well as enhanced experiments that exploit matter effects on the oscillation probabilities $[6,7,8]$.

In the first part of this document, we focus on the measurement of the oscillation parameters $\theta_{23}$ and $\Delta m_{32}^{2}$ presented in [9]. Using a sample of atmospheric high energy neutrinos from $10 \mathrm{GeV}$ to $100 \mathrm{GeV}$ recorded with DeepCore, a precision comparable to leading experiments in the field $[10,11,12]$ is achieved. Further improvements toward becoming competitive with worldleading experiments in this area are expected as analysis techniques are refined and systematic uncertainties are better understood.

Having demonstrated the ability to measure the "atmospheric" mixing parameters at high accuracy with a sparsely instrumented detector located in a natural medium, IceCube is now exploring if it can target the remaining unknowns in the leptonic sector: the ordering of the mass eigenstates, the extent to which Charge-Parity symmetry may be violated in the neutrino sector and wether neutrinos are of Dirac or Majorana nature. The Precision IceCube Next Generation Upgrade (PINGU) experiment, which will be able to reconstruct a high-purity, high-statistics sample of 3-20 GeV atmospheric neutrinos, will be capable of determining this mass ordering. The ordering, or hierarchy, is denoted "normal" $(\mathrm{NH})$ when $v_{3}$ is the most massive of the three neutrino mass eigenstates and "inverted" (IH) if it is the least. This ordering becomes accessible to atmospheric neutrino oscillation experiments via the MSW effect [2,3]: elastic scattering of the $v_{\mathrm{e}}+e^{-} \rightarrow v_{\mathrm{e}}+e^{-}$provides an effective potential for the $v_{\mathrm{e}}$ state as it traverses matter. The effect of this potential on the oscillation probabilities is a parametric enhancement of the oscillation probabilities that depends on the neutrino mass ordering as well as the charge-parity of the neutrinos. While PINGU can not distinguish between neutrinos and anti-neutrinos, which are present in roughly equal amounts in the atmospheric flux, the difference in the neutrino-nucleus cross-section yields an observable signature. The status of the sensitivity study of PINGU to the mass hierarchy, as well as the appearance of $v_{\tau}$ flux not present in the atmospheric flux at production but generated through oscillations, is presented in the second part. 
Finally we conclude with R\&D efforts on optical sensors that allow to detect a higher number of Cherenkov photons from the neutrino interactions and/or their direction, promising a significant performance boost for a future extension of IceCube.

\section{IceCube and DeepCore}

IceCube is an ice Cherenkov neutrino detector located at depths between $1450 \mathrm{~m}$ and $2450 \mathrm{~m}$ at the geographic South Pole. It consists of 5160 downward-facing 10-inch PMTs, enclosed in glass pressure spheres, known as digital optical modules (DOMs) [13]. The detector is an array of 86 strings, each holding 60 DOMs. Of these, 78 strings are arranged in a triangular grid with a typical distance of $125 \mathrm{~m}$ between the strings and a vertical distance of $17 \mathrm{~m}$ between DOMs on the strings. The lower center region of the array, from $1760 \mathrm{~m}$ down to $2450 \mathrm{~m}$, houses a region of denser instrumentation (7 m DOM spacing) known as DeepCore [14] with eight strings at stringto-string distances between $40-70 \mathrm{~m}$. Some $50 \%$ of the PMTs in this region have $35 \%$ higher quantum efficiency than the standard IceCube PMTs. The result is a neutrino energy threshold in DeepCore of about $10 \mathrm{GeV}$, an order of magnitude smaller than in IceCube.

\subsection{Neutrino oscillations with DeepCore}

The IceCube detector records more than $10^{5}$ atmospheric neutrinos every year, a large fraction of them in the DeepCore sub-array [14]. These neutrinos cover path lengths through the Earth ranging from $10 \mathrm{~km}$ to about $12700 \mathrm{~km}$ depending on their arrival zenith angle, $\theta_{z}$. Above $\mathrm{GeV}$ energies they follow a steeply falling spectrum that covers several orders of magnitude up to a few hundred $\mathrm{TeV}$. Neutrino oscillations modify the flavor ratio of the flux of atmospheric neutrinos measured at the detector site. The strongest effect to which DeepCore is sensitive is the disappearance of $v_{\mu}$, modulated by the large (atmospheric) mass splitting, with $\Delta m_{32}^{2} \simeq \Delta m_{31}^{2}$, and the mixing angle $\theta_{23}$ [6]. The best measured values of these parameters $[10,11,12]$ predict a maximum disappearance at $E_{v} \sim 25 \mathrm{GeV}$ for paths that cross the entire Earth $\left(\cos \theta_{z}=-1\right)$, while neutrinos with lower energies reach maximum disappearance at shorter baselines $\left(\cos \theta_{z}>-1\right)$.

Muon neutrino charged current (CC) interactions in the ice with energies between $10 \mathrm{GeV}$ and $100 \mathrm{GeV}$, the primary signal event in this analysis, typically produce a minimum-ionizing muon track and initiate a hadronic shower, while $v_{\mathrm{e}}$ and $v_{\tau}$ charged and neutral current interactions mostly produce shower-like features, with little possibility to distinguish between the later two flavours. A natural classification of events is therefore given by the track-like $\left(v_{\mu}(\mathrm{CC})\right)$ and cascade-like $\left(v_{\mathrm{e}}(\mathrm{CC})\right.$, most $\left.v_{\tau}(\mathrm{CC}), v_{\mathrm{x}}(\mathrm{NC})\right)$ signatures. The detected signal of these interactions in DeepCore are individual Cherenkov photons that are partially scattered due to the optical properties of the ice.

The dominant sources of background for this measurement are muons from cosmic ray showers, as well as confusion of the track- and cascade-like signatures. The event selection, described in detail in [9], has the goal of identifying events that start in the detector volume with a clear muon track to reduce the background. To avoid contamination from atmospheric muons, the data analyzed consists only of events reconstructed as passing through the Earth $\left(\cos \theta_{z} \leq 0\right)$. However, while atmospheric muons enter the detector only from above $\left(\cos \theta_{z} \geq 0\right)$, the small probability of a mis-reconstruction combined with the large number of atmospheric muon events results in a significant contamination of the neutrino sample. Using the outer part of the DeepCore detector 


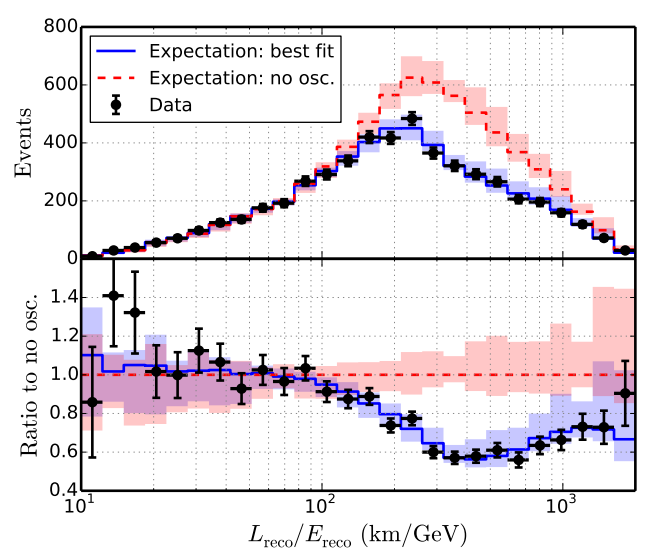

Figure 1: Distribution of events as a function of reconstructed $L / E$ compared to the best fit and expectation with no oscillations (top) and the ratio of data and best fit to the expectation without oscillations (bottom). Bands indicate estimated systematic uncertainties.

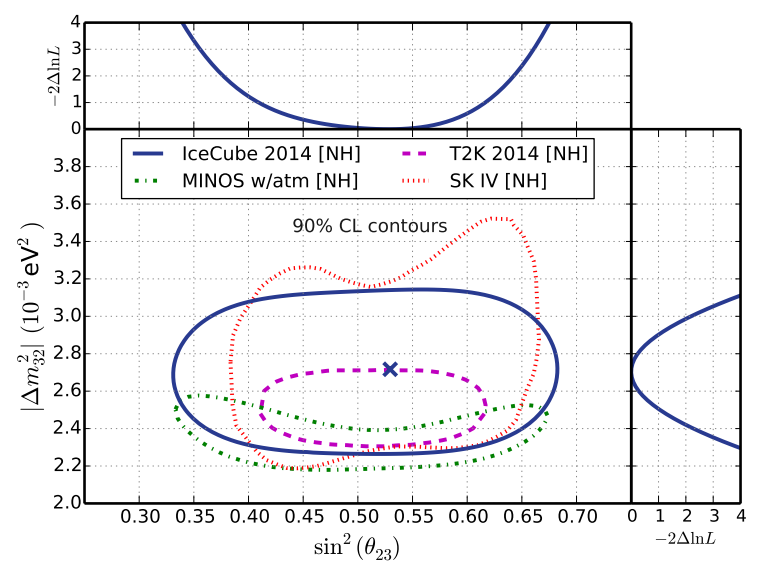

Figure 2: $90 \%$ confidence contours of the result in the $\sin ^{2} \theta_{23}-\Delta m_{32}^{2}$ plane in comparison with the ones of the most sensitive experiments $[11,10,12]$. The loglikelihood profiles for individual oscillation parameters are also shown (right and top). A normal mass ordering is assumed.

as an efficient veto region, a very clean sample of atmospheric neutrinos with $74 \% v_{\mu}(\mathrm{CC}), 13 \%$ $v_{\mathrm{e}}(\mathrm{CC}), 5 \% v_{\tau}(\mathrm{CC})$ and $8 \%$ neutral current interactions is obtained. The expected atmospheric muon contribution to the final sample is less than $5 \%$.

To determine the oscillation parameters, a binned maximum likelihood on a two-dimensional histogram in $\log _{10}\left(E_{\text {reco }}\right)$ and $\cos \left(\theta_{\text {reco }}\right)$ is used, that includes nuisance parameters to account for systematic uncertainties [1]. The physics parameters of the fit are the mixing angle $\theta_{23}$ and the mass splitting $\Delta m_{32}^{2}$. Oscillation probabilities are calculated using a full three-flavor scheme, including the effects of the Earth's matter distribution [15]. The mixing angle $\theta_{13}$ is treated as a nuisance parameter using the constraints from [1]. The remaining oscillation parameters are fixed to the values given in [16], as their uncertainties have negligible impact on the result.

Further nuisance parameters considered here are: the atmospheric neutrino flux, including its normalization $( \pm 20 \%)$ and its spectral index $( \pm 0.04)$, as well as the ratio of $v_{e} / v_{\mu}( \pm 20 \%)$. In addition, cross-section uncertainties are estimated from the GENIE neutrino interaction generator [17], but turn out to be smaller than the atmospheric neutrino flux uncertainties which impact the expectation in a similar way. For example, the effects of the uncertainty on resonant $\left(M_{A}^{R E S}\right)$ and quasi-elastic axial masses $\left(M_{A}^{C C Q E}\right)$ on the cross-section of non-DIS events, $\pm 20 \%$ and ${ }_{-15 \%}^{+25 \%}$ respectively, were tested and found to modify the result by less than $1 \%$.

The analysis was applied to data taken between May 2011 and April 2014, corresponding to 953 days of detector livetime. A total of 5174 events were observed, compared to an expectation of 6830 events assuming no oscillations. The parameters that describe the data best are, for a normal mass ordering, $\sin ^{2} \theta_{23}=0.53_{-0.12}^{+0.09}$ and $\Delta m_{32}^{2}=2.72_{-0.20}^{+0.19} \times 10^{-3} \mathrm{eV}^{2}$, and for an inverted mass ordering, $\sin ^{2} \theta_{23}=0.51_{-0.11}^{+0.09}$ and $\Delta m_{32}^{2}=-2.73_{-0.21}^{+0.18} \times 10^{-3} \mathrm{eV}^{2}$, with no significant preference found for the mass ordering. Statistical and systematic uncertainties have an almost equal contribution to the errors of the final result. The simulation is in good agreement with the data, 
with a $\chi^{2} /$ d.o.f. $=54.9 / 56$ for the 2 -dimensional energy-zenith angle histogram used in the fit. Figure 1 shows the agreement between data and simulation for a projection on reconstructed baseline over energy $\left(L_{\text {reco }} / E_{\text {reco }}\right)$, a variable that does not directly enter the analysis. The distribution of events is peaked close to the first oscillation minimum with a clear deficit due to $v_{\mu}$ disappearance compared to the no-oscillation hypothesis. The $90 \%$ confidence contours on the atmospheric oscillation parameters derived from this analysis, compared to the results from other experiments, are shown in Fig. 2. Even though this measurement is made at significantly higher energies than other experiments and suffers from the uncertainties provided by a natural neutrino source and a detector embedded in a natural medium, the results are compatible and the precision achieved is comparable.

While higher statistics, ongoing improvements in veto algorithms, and the inclusion of cascadelike events will enhance the sensitivity of the oscillation studies with IceCube in the near future, the event statistics at low energies to achieve compelling sensitivity to the neutrino mass hierarchy can only be reached by deploying additional instrumentation within the DeepCore array. This will allow to collect more light per event and thus not only increase the statistics below $E_{v}=10 \mathrm{GeV}$, but also improve the energy and directional reconstruction.

\section{PINGU}

The design for the PINGU detector (see Figure 3) follows closely that of DeepCore with the new modules being deployed at the center of the IceCube strings in the known clearest region of the ice. With an average $22 \mathrm{~m}$ horizontal string-to-string spacing and $3 \mathrm{~m}$ vertical sensor-to-sensor spacing, the PINGU modules will have a significantly increased density which lowers the energy threshold for the array from $10 \mathrm{GeV}$ to a few GeV. PINGU's location within the IceCube and DeepCore detector arrays allows to extend the current active veto applied for atmospheric muons and thereby enhance the background rejection [14]. In contrast to the geometry described in [18], meanwhile we have optimized the horizontal and vertical spacing of the optical sensors in

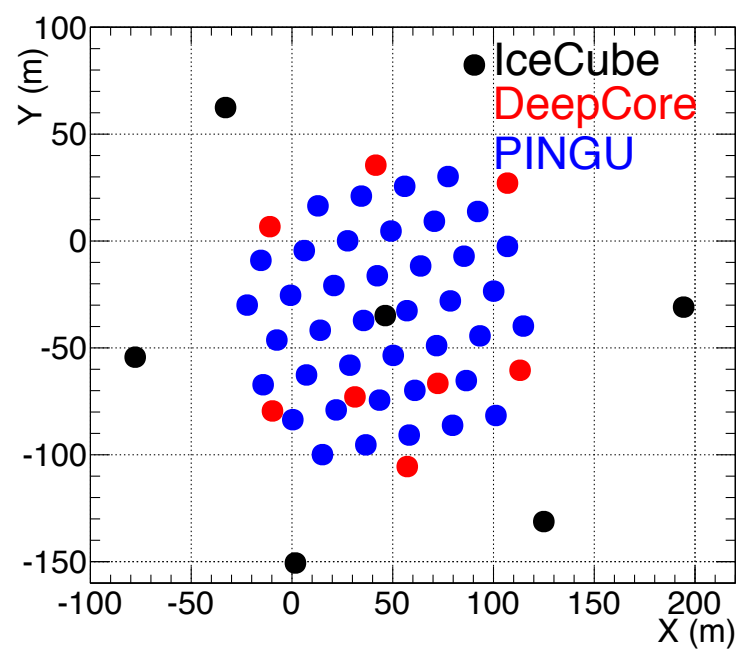

Figure 3: A top view of the proposed PINGU detector geometry used for simulation optimization studies. The location of existing IceCube strings are shown in black, DeepCore in red and PINGU in blue the detector to achieve the highest sensitivity to the oscillation physics, most notably a measurement of the mass hierarchy, assuming the atmospheric neutrino signal-to-background levels achieved are comparable with those in DeepCore.

\subsection{Neutrino mass hierarchy}

In addition to the vacuum oscillations already measured by DeepCore, there are two distinct physical effects that play a role in the oscillations as neutrinos propagate through the earth. The first 

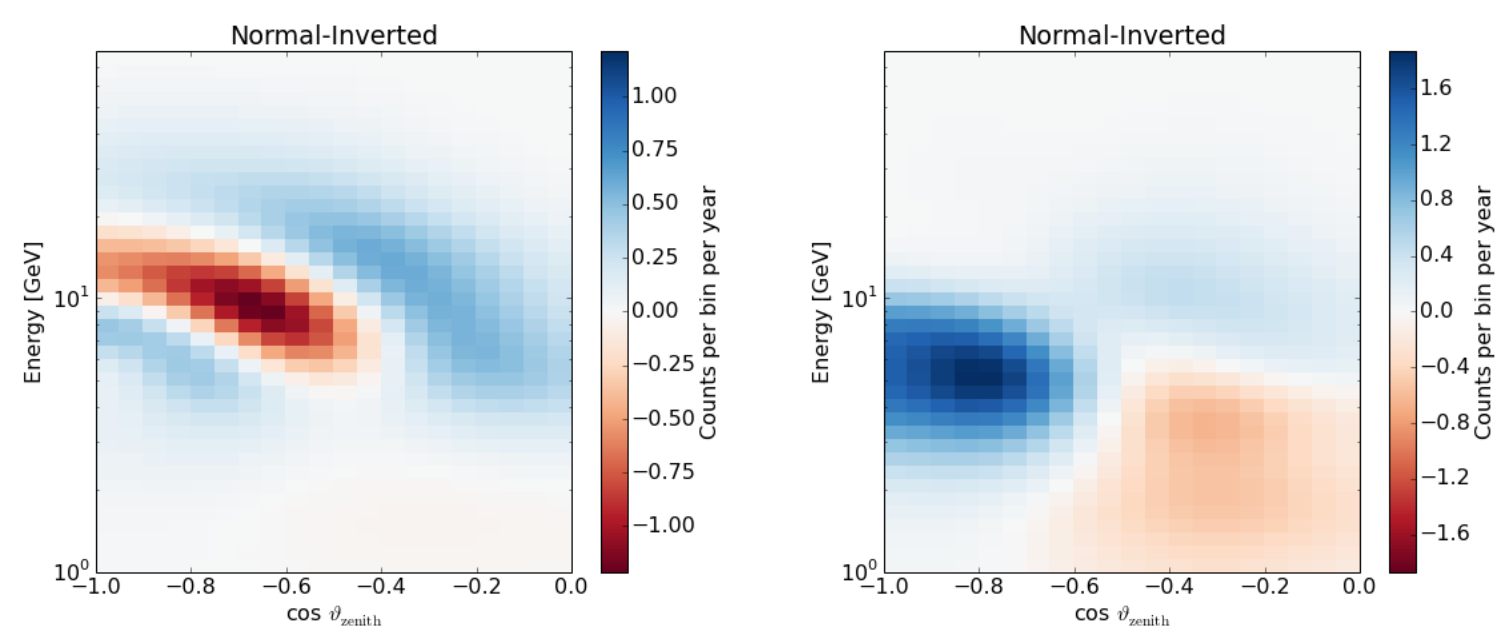

Figure 4: Difference in event counts per year after rejection cuts between the normal and the inverted hierarchy for track-like (left) and cascade-like (right) events using the updated geometry shown in Fig. 3.

is the MSW effect $[2,3]$ that results in an enhancement of the oscillation probability for $v_{\mu} \rightarrow v_{e}$ $(\mathrm{NH})$, or $\bar{v}_{\mu} \rightarrow \bar{v}_{e}(\mathrm{IH})$. It is strongly dependent on the matter density for along path through the Earth. The second effect arises from the density transition at the Earth's mantle-core interface [15] where neutrinos passing through this interface can undergo "parametric enhancement" of their oscillation probability [7].

The aggregate effect of these phenomena on muon neutrinos, in both the $\mathrm{NH}$ and $\mathrm{IH}$, scenarios is that the survival probabilities of anti-neutrinos in the $\mathrm{NH}$ are essentially identical to that for neutrinos in the IH, and vice versa. Precision measurement of the survival probabilities in the energy range targeted by PINGU in the presence of these matter effects would thus permit a determination of the neutrino mass hierarchy (NMH). However, the atmospheric flux that will be detected by PINGU consists of approximately equal numbers of neutrinos and anti-neutrinos, making discrimination between the hierarchies more challenging. Yet, the asymmetries in the cross sections and kinematics of $v$ and $\bar{v}$ interactions with nuclei lead to different detected event rates in the detector, so that a measurement can still be made even without explicit $v-\bar{v}$ discrimination [19].

The effect is strongest for neutrinos crossing the Earth's core, i.e. at $-1.0<\cos \left(\theta_{z}\right)<-0.8$ in the energy range between $3-15 \mathrm{GeV}$. While the initial feature amounts to a $\pm 10 \%$ effect in this sensitive region, it is significantly diluted by the limited angular and energy resolution of the detector. Figure 4 shows the difference between the number of detected track- and cascade-like events per year under each hierarchy binned as a function of the reconstructed neutrino energy, $\mathrm{E}_{v}$, and the cosine of the reconstructed zenith angle, $\cos \vartheta_{z}$. Still one find regions in which the number of events expected for the $\mathrm{NH}$ is greater than that expected for the $\mathrm{IH}$ (blue regions) and vice-versa (red regions) by about $1 \%$ in some bins. Sensitivity to this pattern of the event number differences as a function of $\mathrm{E}_{v}$ and $\cos \vartheta_{z}$ permits one to determine the neutrino mass hierarchy. The size of the observable effect clearly demonstrates the need for a high statistics data sample with very good control of the systematic uncertainties. 


\subsection{Sensitivity estimate}

Figure 5 shows the median significance that PINGU will obtain for the neutrino mass hierarchy as a function of detector livetime from an initial study [18] using a smaller geometry. In the meantime, a number of improvements have been made to this analysis, that will be briefly described in the following.

While it was found that the initial analysis suffered from an incomplete description of the self-noise of the optical modules, and while correcting this effect worsened the obtainable angular and energy resolution of the events, an improved background rejection and an improved particle classification at the same time increased the signal efficiency of the analysis, resulting in a small net effect.

More notably, significant improvement has been made in the evaluation of systematic parameters and the statistical methods used to arrive at the final result. The analysis presented in Fig. 5 takes into account the relevant oscillation pa-

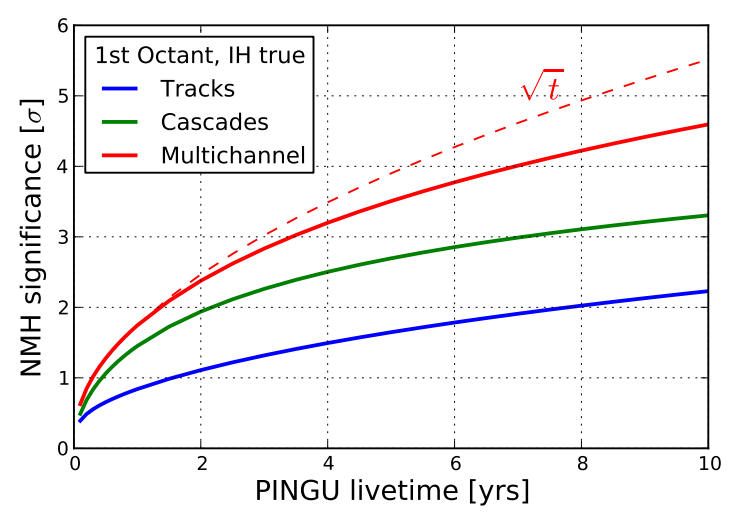

Figure 5: The PINGU significance to the neutrino mass hierarchy as a function of time, using the analysis in [18] and considering full complement of systematics. For reference, the red dashed line shows the expectation for a $\sqrt{t}$ dependence. rameters as well as effective parameters describing errors in the energy scale and scale factors for the overall flux / effective area as well as independent scale factors for neutrino and anti-neutrino cross-sections (with some degeneracy among the latter). We have now implemented physical uncertainties on the neutrino-nucleon interactions following the description in [17], including uncertainties on the axial mass for chargedcurrent quasi-elastic $M_{A}^{C C Q E}$ and resonant $M_{A}^{R E S}$ production, as well as the Bodek-Yang highertwist parameters $A_{H T}^{B Y}, B_{H T}^{B Y}, C_{V 1 u}^{B Y}$ and $C_{V 2 u}^{B Y}$. Figure 6 shows that the reduction of sensitivity from marginalizing over these additional systematic parameters is small and that they had been well accounted for in our effective description used in [18].

The computational challenge of minimizing over a multi-dimensional parameters space in combination with the requirement to calculate the individual steps of the experimental outcome (flux, oscillations and detector response) at a higher precision than the small effect of the mass hierarchy had initially limited us to using a $\Delta \chi^{2}$-based approach based on the Fisher information matrix to obtain the sensitivity [18]. Through an improved minimizing method as well as parallelization of the oscillations probability calculation on GPUs, we have now significantly sped up the approach to allow individual likelihood minimizations of an ensemble of pseudo-experiments. Both methods have been shown to agree for the set of systematics implemented in [18].

While the proper treatment of noise in the optical sensors and the inclusion of additional systematic parameters diminish the observable NMH signature, the optimized geometry, event selection and improved track vs. cascade classification improve the situation. The overall result is a NMH significance that is not very different from the situation described in [18] and shown in 


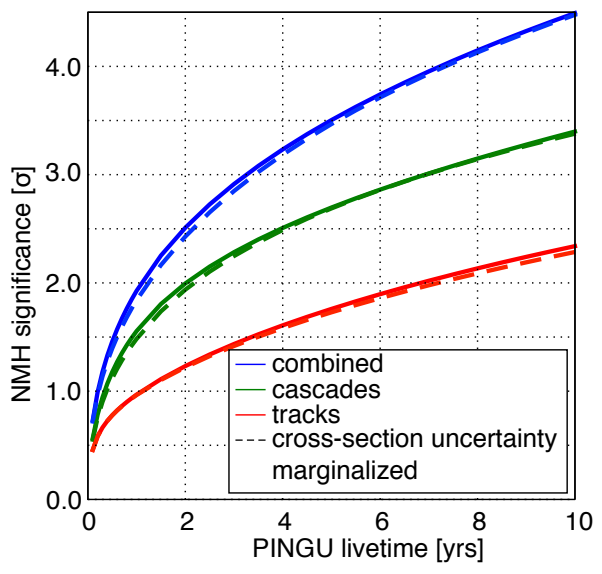

Figure 6: PINGU sensitivity to the NMH vs livetime without (full) and with (dashed) marginalizing over the uncertainties in the $v N$ cross-section

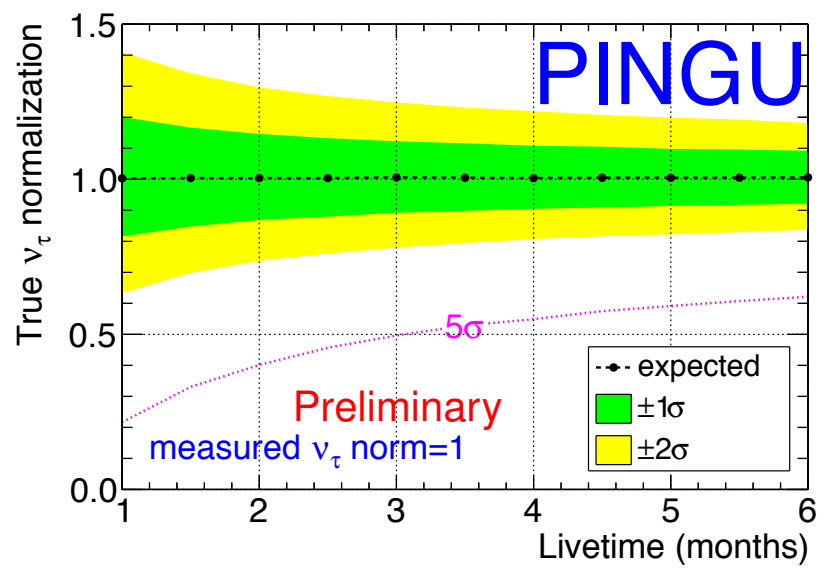

Figure 7: PINGU Sensitivity to $v_{\tau}$ appearance as a function of detector livetime. The green (yellow) bands give the $1 \sigma(2 \sigma)$ error on the measured $v_{\tau}$ flux if it has its nominal value. The relative $v_{\tau}$ flux below the dotted line can be excluded at $5 \sigma$

Figure 5, but with significantly increased accuracy and confidence in the projected sensitivity.

\section{3 $v_{\tau}$ appearance}

Complementing the $v_{\mu}$ disappearance measurement with DeepCore described above, PINGU will also have the capability to perform a measurement of $v_{\tau}$ appearance. Both Super-Kamiokande [20] and OPERA [21] have completed studies for $v_{\tau}$ appearance, confirmed independently to about $4 \sigma$. While it is very likely the $v_{\tau}$ events are indeed appearing from $v_{\mu}$ oscillations, we may learn more about neutrino oscillations by comparing the $v_{\tau}$ appearance rate to that expected from $v_{\mu}$ disappearance. If those rates are not compatible we would have an indication of new physics either from non-unitary of the lepton mixing matrix (indicating the presence of sterile neutrinos) or from non-standard interactions. A first study on $v_{\tau}$ appearance with PINGU has been performed using the same selection criteria as the NMH analysis to reject atmospheric muons[18], while selecting a purer sample classified as cascade-like events. In that sample we expect roughly $1.7 \mathrm{k} v_{\tau}$-induced events per year on a background of $11.2 \mathrm{k}$ events. Here the signal is concentrated around the upgoing region, while the background is concentrated around the horizon. Even though there is more background than signal in the up-going region, the measurement of $v_{\tau}$ appearance remains statistically significant. The analysis was performed assuming a similar set of systematic errors as for the NMH analysis and yields an expected $5 \sigma$ exclusion of the no $v_{\tau}$ appearance hypothesis with 1 month of data and $10 \%$ precision on the $v_{\tau}$ normalization after 6 months (see Figure 7).

\section{Optical sensors}

For all studies described above, the photon effective area and angular acceptance of the optical sensors is assumed to be identical to those of the higher quantum-efficiency version of the IceCube DOM currently used in parts of the DeepCore subarray. In fact, the baseline optical sensor design 


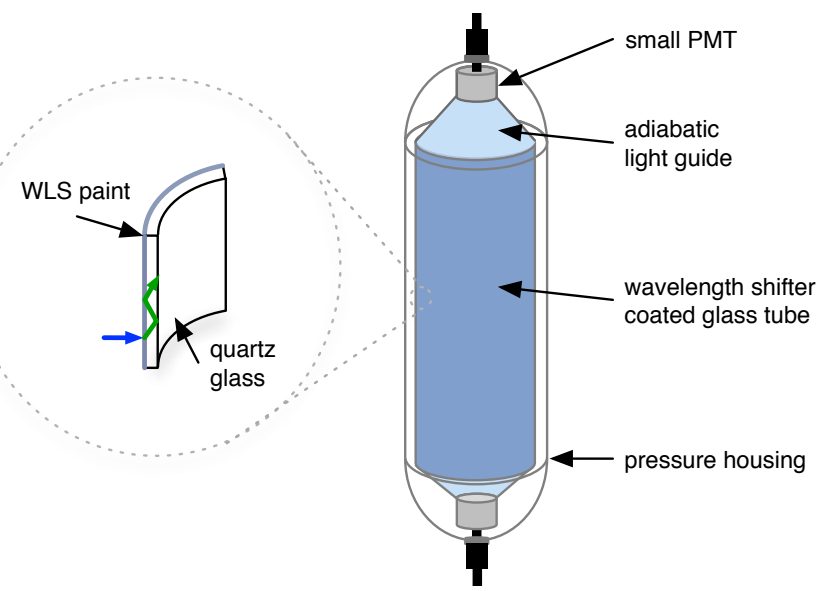

Figure 8: Sketch of the wavelength-shifting optical module (WOM)

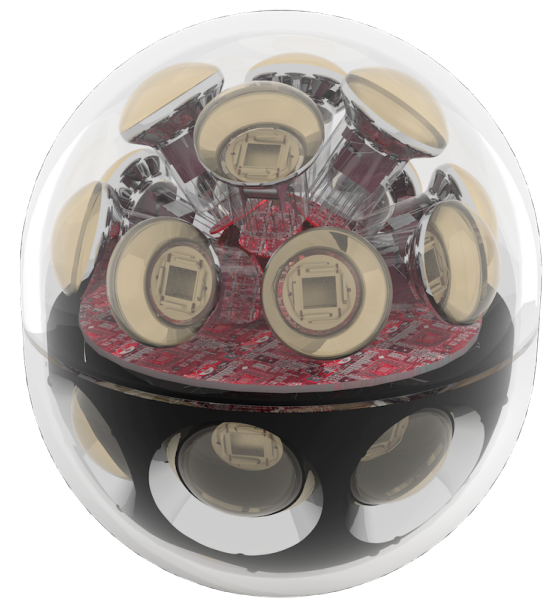

Figure 9: Sketch of the multi-PMT optical module (mDOM)

for PINGU - the Gen2-DOM - is an updated version of the IceCube DOMs with minimal changes to the mechanical design but rigorously modernized electronics [22]. In addition, alternative types of optical modules are under development. The focus of these developments is to increase the photon collection area, minimize the noise, and improve the time and directional resolution. The installation of PINGU will allow us to co-deploy a selected set of new photon detection instruments, and subsequently calibrate and test them using the IceCube and PINGU infrastructures. This enables detailed studies of the in-situ performance of these devices and their suitability for the High Energy Array [22] of the IceCube-Gen2 facility or a future megaton-scale detector with sensitivity to events at and below the $\mathrm{GeV}$ energy scale, providing e.g. sensitivity to proton decay and bursts of supernova neutrinos from nearby galaxies [23]. Taking advantage of the excellent properties of Antarctic ice: low temperature, low radioactivity, and little absorption above UV wavelengths of $200 \mathrm{~nm}$, the R\&D efforts so far focus on two major directions:

- low noise, UV-sensitive, wavelength-shifting modules (WOMs) with large area but relaxed timing requirements

- multi-PMT optical modules (mDOMs) with increased photodetection sensitivity, directional information, and precision timing

WOMs: The essential idea of the WOM is to increase the sensitive area of a PMT by passive components that act as light collectors and concentrators as illustrated in Fig. 8. The goal is to maximize the collection area for cigar-shaped modules that safely withstand high pressure, can be handled easily and may be produced at a price per photosensitive area that is lower than that for standard optical modules. At a larger photo-detection efficiency, the resulting geometry also provides a more elongated module that has a significantly smaller diameter, which may allow to decrease the drill hole size in future installations and thus yield further significant cost reduction. mDOMs: In contrast, multi-PMT optical modules, first developed for the KM3NeT neutrino telescope in the Mediterranean Sea [24], house arrays of several small PMTs and their read-out electronics. Such modules offer a number of attractive advantages compared to the conventional layout 
such as an increased photocathode area with almost $4 \pi$ acceptance, local coincidence recognition and an improved event reconstruction and background suppression due to directional sensitivity for the photons. The KM3NeT multi-PMT concept has been adapted for IceCube by incorporating 24 three-inch PMTs in a 14-inch spherical glass housing with a short cylindrical extension suitable for deployment in holes achievable with the IceCube drill equipment. The goal is to achieve an overall price per effective photon area at least as low as for conventional optical modules.

While both approaches provide an enhanced photo detection sensitivity, the low noise and UV-sensitivity of the WOM approach mostly promises a reduced energy threshold and energy resolution. In contrast, the directional information and precise timing of the mDOMs may prove particularly beneficial for the event reconstruction accuracy. To exploit the complementarity of the technologies, both types of modules may eventually be included in the detector.

\section{References}

[1] K. A. Olive et al. (Particle Data Group), Chin. Phys. C38, 090001 (2014)

[2] L. Wolfenstein, Phys. Rev. D17 2369-2374 (1978)

[3] S. Mikheev, A. Smirnov, Soviet Journal of Nuclear Physics 42 913-917 (1985)

[4] Y. Fukuda et al. (Super-Kamiokande Coll.), Phys. Rev. Lett. 81 1562ff (1998)

[5] M. C. Gonzalez-Garcia, M. Maltoni and T. Schwetz, JHEP 1411 (2014) 052 [arXiv: 1409.5439 ]

[6] E. Fernandez-Martinez, et al., Phys. Rev. D 82 (2010) 093011 [arXiv: 1008 . 4783]

[7] E. K. Akhmedov, S. Razzaque and A. Y. Smirnov, JHEP 1302 (2013) 082 [arXiv: 1205 . 7071]

[8] W. Winter, Phys. Rev. D 88 (2013) 1, 013013 [arXiv: 1305 . 5539]

[9] M. G. Aartsen et al. (IceCube Coll.), Phys. Rev. D 91 (2015) 7, 072004 [arXiv: 1410 . 7227]

[10] P. Adamson et al. (MINOS Coll.), Phys. Rev. Lett. 110, 251801 (2013), [arXiv/1304 . 6335]

[11] K. Abe et al. (T2K Coll.), Phys. Rev. Lett. 112, 181801 (2014), [arXiv/1403.1532]

[12] A. Himmel (Super-Kamiokande Coll.), 7th International Conference on Interconnection between Particle Physics and Cosmology (PPC 2013) (2013) [arXiv/1310 . 6677]

[13] R. Abbasi et al. (IceCube Coll.), Nucl. Instrum. Meth. A 601 (2009) 294 [arXiv : 0810 . 4930]

[14] R. Abbasi et al. (IceCube Coll.), Astropart. Phys. 35 (2012) 615 [arXiv: 1109 . 6096]

[15] A. M. Dziewonski, D. L. Anderson, Physics of the Earth and Planetary Interiors, 25, 297 (1981)

[16] F. Capozzi, G. L. Fogli, E. Lisi, et al., J. Phys. Conf. Ser. 598 (2015) 1, 012002

[17] C. Andreopoulos, et al., Nucl. Instrum. Meth. A 614 (2010) 87 [arXiv: 0905.2517]

[18] M. G. Aartsen et al. (PINGU-IceCube Coll.), [arXiv:1401.2046v1]

[19] O. Mena, I. Mocioiu and S. Razzaque, Phys. Rev. D 78 (2008) 093003 [arXiv: 0803.3044 ]

[20] M. Nakahata et al. (Super-Kamiokande Coll.), these proceedings

[21] A. Paoloni et al., (OPERA Coll.), these proceedings

[22] M. G. Aartsen et al. (IceCube Coll.), [arXiv:1412.5106]

[23] S. Böser, M. Kowalski, L. Schulte, et al., Astropart. Phys. 62 (2015) 54 [arXiv: 1304 . 2553]

[24] S. Adrian-Martinez et al. (KM3NeT Coll.), Eur.Phys.J. C74 (2014) 3056, [arXiv: 1405 . 0839] 